\title{
Proyectos de extensión como promotores de un aprendizaje significativo para alumnos de Ciencias Veterinarias en la Universidad Nacional de Rosario
}

\author{
Renato Biolatto \\ rebiolatto@gmail.com
}

\section{Raúl Vallone \\ rvallonevet@hotmail.com}

Carla Vallone

carla.p.vallone@gamil.com
Andrés Bassi

andresbassi@hotmail.com

Docentes investigadores de la Facultad de Ciencias Veterinarias. Universidad Nacional de Rosario, Argentina.
Integración de la docencia y la extensión /

Intervenciones

RECEPCIÓN: 24/06/16

ACEPTACIÓN FINAL: 10/10/16

\section{Resumen}

Conocemos a necesidad de articular la universidad con la sociedad y sabemos del valor que las prácticas educativas solidarias, como voluntariado, práctica preprofesional o aprendizaje servicio tienen sobre la formación de futuros profesionales.

Si bien en la Universidad Nacional de Rosario (UNR), la extensión ha cobrando vigencia en cantidad y calidad de proyectos, los mismos, comparados con los de investigación, son escasos y con poca participación estudiantil. En la convocatoria 2015 de la Secretaría de Extensión UNR, en Ciencias Veterinarias no se alcanzó siquiera a cubrir el cupo asignado de proyectos a subsidiar. El diseño curricular no prevé la incorporación de experiencias de extensión en articulación con las asignaturas de cursado regular. No obstante, experiencias como la que se ha de relatar dan cuenta del valor de la formación social y de la necesidad de colocar a los alumnos en situaciones reales, distintas de las abordadas en el aula.

Palabras-clave

- Extensión

- Participación

- Alumnos

- Formación

- Social

\section{Resumo}

Conhecemos a necessidade de articular a Universidade com a sociedade, e sabemos o valor que as práticas educativas solidárias como o voluntariado, prática pré-profissional ou aprendizagem serviço têm na formação dos futuros profissionais.

Embora na Universidade Nacional de Rosario (UNR) a extensão fosse ganhando força em quantidade e qualidade dos projetos, comparando-os com os de pesquisa, são escassos e com pouca participação dos alunos. A convocação de 2015 da Secretaria de Extensão da UNR, em Ciências Veterinárias, não chegou nem a cobrir a cota reservada para projetos a subsidiar.

O currículo não prevê a incorporação de experiências de extensão em articulação com as disciplinas do curso regular. Contudo, experiências como a relatada demonstra o valor da formação social e a necessidade de colocar os alunos em situações reais, diferentes das aboradadas em sala de aula.

Palavras-chave

- Extensão

- Participação

- Alunos

- Formação

- Socia
Para citación de este artículo

Biolatto, R.; Vallone R.; Vallone, C.; Bassi, A. (2016). Proyectos de extensión como promotores de un aprendizaje significativo para alumnos de Ciencias Veterinarias en la Universidad Nacional de Rosario, Argentina. En Revista +E versión digital, (6), pp. 360-369. Santa Fe, Argentina: Ediciones UNL. 


\section{La Universidad Nacional de Rosario y la extensión} Dasso Lucrecia (2011), siguiendo a distintos autores, explicita que, si bien es a partir de la Reforma Universitaria de 1918 que la problemática de la extensión se afirma como un factor indispensable para el logro de la autonomía e integración social de las universidades con la sociedad, esto no siempre ha sido posible de ser llevado adelante. Más de 90 años pasaron desde aquella Reforma y muchos momentos les han tocado vivir a las universidades públicas, en los cuales se pusieron en tela de juicio los principios reformistas (Chiroleu y otros, 2001).

Dable es reconocer que la extensión hacia sectores sociales de menores recursos se convierte en la herramienta más importante que tienen las universidades para afrontar las crisis sociales, y especialmente Argentina en estos momentos. ${ }^{1}$

Pero se hace necesario definir la "extensión" y afrontar el inevitable dilema que se presenta cuando se tratan de cubrir todos los aspectos de un proceso institucional y social tan complejo. Leonor Perez Zaballa (2007) manifiesta que la extensión contribuye a la formación de los ciudadanos y a un desarrollo sostenible, equitativo y respetuoso de los derechos del hombre. Se busca alcanzar un concepto de extensión universitaria que se aleje del modelo economicista de prestación de servicios y del modelo asistencialista de proyectos comunitarios para definir así una función que permita a la universidad interactuar proactivamente con su entorno social. De este modo, la extensión se erige como una dimensión más del proceso educativo que se articula transversalmente con las restantes funciones de la universidad, promoviendo intra y extramuros cultura, ciencia, técnica y valores que comprometen al hombre con la sociedad.
Yamile Socolovsky analiza la extensión en su carácter de "Práctica Social Comunitaria" (PSC) y señala que se precisa

"conceptualizar estas prácticas en términos que permitan superar las connotaciones que implican el discurso de la extensión, signado por la visión de una irreductible exterioridad de la universidad respecto a la sociedad" (2015:12).

Reconocemos que "las universidades normalmente están más acostumbradas a dirigir su mirada hacia adentro de ellas mismas que hacia la sociedad que hace sus reclamos" (Biolatto, 2011:26).

\section{La extensión hacia el interior de la Facultad de Ciencias Veterinarias}

Contreras (1997) puso de manifiesto que el saber sistemático y la actitud de servicio de las profesiones son una "función social crucial". Sabemos que el perfil profesional adquirido por un egresado universitario es el resultado de múltiples acciones que interactúan durante todo el tiempo de formación. Así el curriculum determina el valor el saber específico, el objeto de estudio y el perfil aspirado por la institución, los que habrán de orientar la formación de los futuros profesionales.

Cabría preguntase: ¿cómo se posicionan los médicos veterinarios ante este compromiso?

\subsection{Haciendo un poco de historia}

Biolatto (2005), al analizar el devenir histórico de las Facultades de Ciencias Veterinarias de Argentina, sostuvo que ellas se originaron partiendo del modelo de universidad francesa, el que se caracterizó
1) Alicia de Alba (2004) sostenía se estaba ante una "Crisis Estructural Generalizada" que tocaba todos los estamentos de una sociedad y que, aunque daba por tierra con los paradigmas que habían guiado las acciones político-sociales, también posibilitaba la construcción conjunta de nuevos paradigmas. 
por la fragmentación del saber en distintas disciplinas. De este modo, y a semejanza de lo acontecido en la Universidad de La Plata, los diseños curriculares fijaron como objeto de estudio al animal en sí mismo y principalmente al animal enfermo, desconociendo o haciendo caso omiso a todo lo que lo rodea, es decir lo contextual, en donde es imposible no pensar en el hombre y la sociedad. Consideramos que ese hacer profesional sesgado por la visión clínica está instaurado desde hace más de dos décadas. En tanto, organizaciones que nuclean a las Facultades de Ciencias Veterinarias del país proponen cambiar el objeto de estudio que orienta los diseños curriculares.

En los albores de los años 90 la idea de cambio de la mirada profesional cobró fuerza, y en la Primera reunión de Escuelas y Facultades de Medicina Veterinaria de América Latina realizada en Baja California se resumieron en un documento, firmado por Oscar Reyes Sánchez y Alejandro Plascencia Jorquera (1993), las principales estrategias que había de tener en cuenta la educación veterinaria para adecuarse a la vinculación con sectores productivos y sociales, la búsqueda de nuevas formas de organización y la intención de resolver los problemas de la sociedad, las que se tornaron en líneas principales de acción.

\subsection{Cambio de paradigma}

Rosemberg y Casas Olascoaga sostenían que, entre los profesionales veterinarios:

"[el] apego al paradigma médico parece establecer límites que impiden ofrecer el desafío de un salto de calidad ante el temor de 'descaracterizar' la profesión veterinaria (...). La búsqueda de un paradigma veterinario debe comenzar por resolver su objeto formal abstracto. Este es, y siempre fue, a nuestro juicio, las poblaciones humanas y no las animales. El objeto social del veterinario debe quedar claramente establecido: el veterinario debe asumir como compromiso y responsabilidad la organización científica y tecnológica de la explotación de los animales para beneficio del hombre". (1991:342-343)

Pese a los avances en las perspectivas curriculares y en la apertura del campo de acción del médico veterinario, actualmente el desempeño profesional en general se focaliza en la dimensión clínica orientada a la atención de pequeños animales. Práctica que frecuentemente absorbe a los noveles veterinarios y que además se desarrolla principalmente en grandes centros urbanos, en detrimento de los sectores agropecuarios.

La extensión en la Facultad en relación con el medio rural es escasa. Un dato que nos muestra la cuantía de la desconexión con el medio es la presentación de proyectos de este tipo en las tres primeras convocatorias que realizó la Secretaría de Extensión de la UNR. Para la convocatoria 2008, sobre 45 proyectos presentados, 4 correspondían a Ciencias Veterinarias; en 2009, sobre 57, 5; en 2010, sobre 58, 3; y en 2015, solamente 3 de los 5 factibles de ser subsidiados. De igual forma, es evidente el bajo número total de proyectos de extensión presentados al subsidio en una de las principales universidades nacionales que cuenta con 12 Facultades.

Cabría entonces hacer una segunda pregunta: ¿ante los cambios acontecidos en el ámbito agropecuario, que han orientado el hacer de este sector colocándolo bajo una lógica netamente mercantilista, desconociendo las implicancias ambientales y sociales, los médicos veterinarios pueden no estar comprometidos ante estos desafíos y demandas sociales actuales?

La respuesta no debería ser otra que una afirmación en el sentido de que, aunque algunas áreas sean compartidas, ellos, junto a otros profesionales de las Ciencias Agropecuarias, son quienes deben buscar, analizar y aplicar soluciones a los reclamos de aquellos sectores que han visto desplazada su participación e inclusión en la producción agropecuaria.

Consecuentemente, no es desde un perfil profesional clásico, encauzado desde lo sanitario mayoritariamente, desde donde los egresados veterinarios han de trabajar para revertir situaciones económico-sociales netamente desfavorables.

Formar estos profesionales implica una nueva manera de transitar el diseño curricular, y la Educación Experiencial” es quizás la metodología más adecuada. "La misma comprende modalidades diversas, como ser: el voluntariado, el aprendizaje-servicio y la práctica preprofesional” (Camilloni, 2013:17). Esta autora también nos aclara que aprendizaje-servicio es una estrategia de enseñanza que contribuye a la educación para la ciudadanía y a la formación ética de los estudiantes. Sobre el particular, María Nieves Tapia (2010:60), siguiendo a otros autores, indica que "siempre es una actividad asociada con una práctica social".

Por lo tanto, una metodología de aprendizaje-servicio se convierte en una herramienta por demás adecuada, ya que en esta actividad educativa los estudiantes conjugan los conocimientos adquiridos poniéndolos en juego en situaciones que requieren de los mismos a fin de cubrir servicios pensados para y por la comunidad en función de valores ciudadanos. Lo que implica un aprendizaje riguroso unido a una acción solidaria planificada. De esta manera, todos aquellos conocimientos adquiridos en al aula se ven enriquecidos al ser llevados a una situación social real y al articularlos dialógicamente con los saberes de los propios actores sociales para lograr la superación de un obstáculo. Se configura una experiencia en la que los propios alumnos toman conciencia de la relevancia de otros tipos de saberes y del valor de la interacción con la sociedad.

Sobre este mismo aspecto, Fabio Erreguerena y otros aportan que en esta experiencia:

"de la visión de un saber disciplinar se pasa a la concepción de

un conocimiento múltiple, complejo, cuya singularidad reside en 


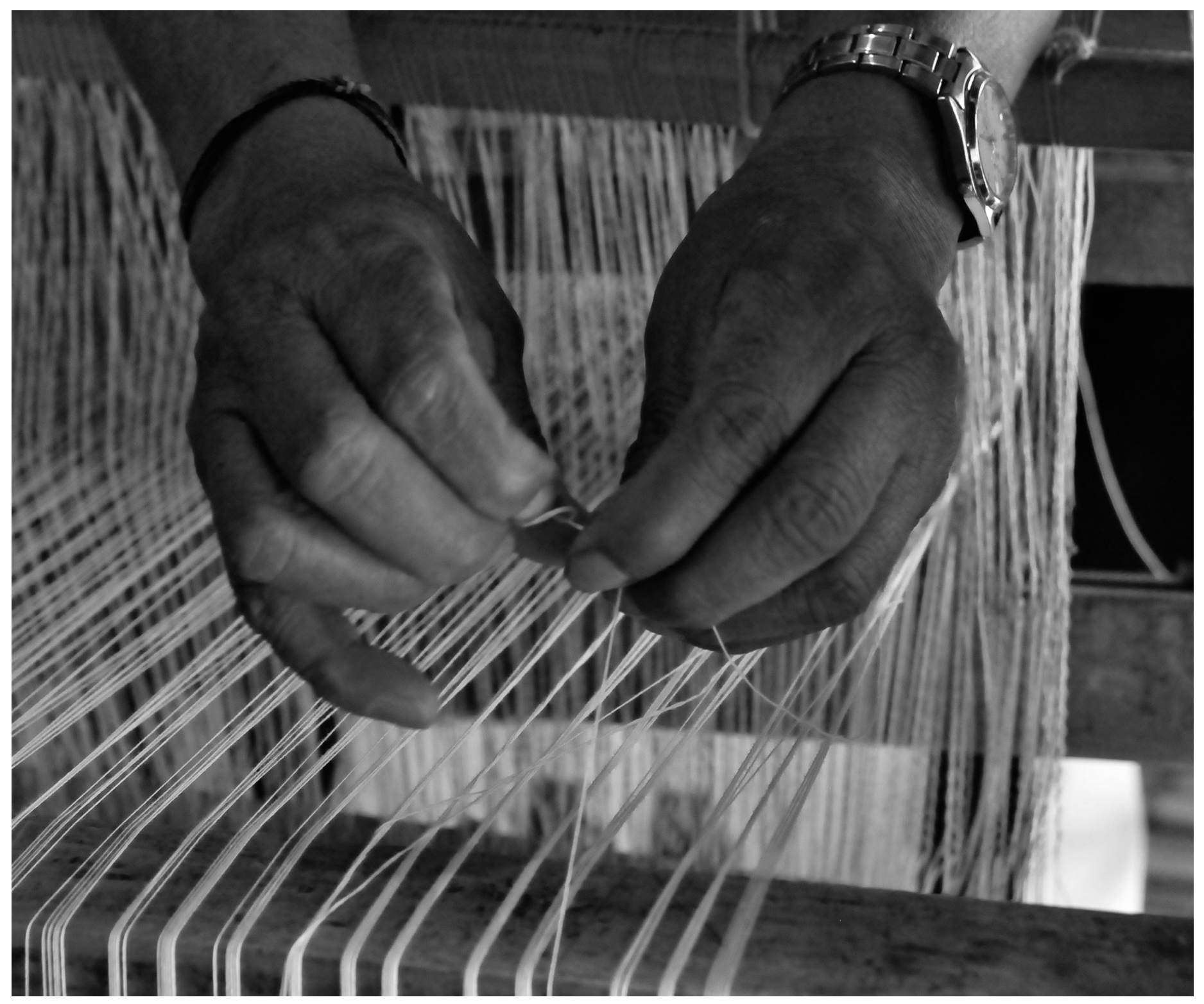

(๑) Oscar Dechiara 
que la universidad ya no tiene el monopolio de su producción, y en que se rompe con la asimetría y relaciones de poder entre conocimiento académico y el resto de los saberes". (2015:61)

\section{Una propuesta de extensión como práctica integradora en la Facultad de Ciencias Veterinarias}

Como equipo integrado por varias cátedras con relación a la Producción Animal, nos interesamos por la problemática socioeconómica de pequeños productores lecheros y/o ganaderos que permanecen en el sector productivo pese a los avatares económicos del sistema que trata de expulsarlos, tanto en el área de influencia próxima a la Facultad como en la localidad de El Colorado, en la provincia de Formosa. Por ser docentes universitarios, consideramos impostergable sumar alumnos en trabajos de investigación-extensión de fuerte contenido e impacto social, pues creemos que así se están promoviendo cambios en la forma de pensar y de actuar profesional de todos estos integrantes. Consecuentemente con esta meta, aspiramos a:

- Establecer juntamente con las familias de pequeños productores lecheros de escasos recursos planes de acción que traten de retenerlas en la actividad productiva.

- Fortalecer estas líneas de trabajo de acción social que se presentan como un caso directo de apertura al medio de la Facultad (de extensión en un sentido cualitativo) e integración con otras instituciones

- Formar recursos humanos ya sea a través de los alumnos becarios directos del proyecto como de los docentes de otras cátedras que han ido sumándose a él.

- Promover cambios en el desarrollo del diseño curricular que se traduzcan en el hacer profesional.

Para alcanzar estos fines apostamos a todo aquel hacer vinculado con la reflexión y la toma de conciencia tendiente a generar acciones en la búsqueda de alternativas superadoras económicas a una situación conflictiva.

Dada la naturaleza participativa que sostiene el presente trabajo, nos permitimos ubicarlo dentro de una perspectiva dialéctica. Ésta hace referencia a las relaciones que se establecen, en todo proceso de investigación/extensión, entre el investigador/ extensionista y aquella parcela de la realidad a investigar y/o sobre la que se ha de actuar.

Se posibilitó un abordaje a la problemática detectada y a la búsqueda de distintas y posibles soluciones, tanto de manejo como de producción y formación. De este modo, el trabajo multidisciplinar e interinstitucional coadyuvó al logro de los objetivos planteados para encontrar soluciones tendientes a tratar la problemática socioeconómica de los pequeños productores lecheros.

Se articuló con la Agencia de Extensión INTA de El Colorado, Escuela Agropecuaria con Pedagogía Alternativa ${ }^{\circ} 4, \mathrm{Km} 210$, Escuela Agropecuaria n ${ }^{\circ} 48$ "El Alba", Cooperativa El Progreso de Río Navegación Bermejo, Km 210, grupos de Cambio Rural y productores independientes.

Esta presentación aborda el impacto del proyecto de extensión sobre los estudiantes universitarios participantes y solo sucintamente se da cuenta de la metodología que enmarcó lo realizado en territorio.

Como se indicó ya en esta presentación, la acción que propusimos constituye una estrategia de conocimiento y apertura de un proceso de desarrollo, con la utilización de experiencias productivas como elementos configuradores de la misma. La realización de un diagnóstico provisional participativo de la realidad en territorio por todos los participantes, en concomitancia con los actores sociales, dio lugar a un Grupo Motor (GM) integrado por técnicos, instituciones, productores, alumnos y demás participantes institucionales vinculados. Esto constituyó una instancia intermedia de análisis y diagnóstico para pasar luego al tercer momento planificado, que implicó el desarrollo de los GM en Proyectos de Acción Participativa vitales para llevar adelante las propuestas superadoras de las problemáticas detectadas conjuntamente (ya sea en el ámbito de la producción, la formación, entre otros). Se definieron los tiempos locales para ejecutar las acciones y se priorizaron las coyunturas de acción intentando una conexión con las instituciones que pudieran brindar apoyo para llevar a cabo la planificación real (Municipalidad, Secretaría de Desarrollo Familiar, entre otras).

\section{Lo hecho en palabras de nuestros alumnos}

En cuanto a la formación de recursos humanos, la propuesta permitió a los alumnos universitarios participantes una formación académica distinta a la alcanzada solo en el ámbito áulico, convirtiéndose así en un lugar donde conjugar la teoría con la práctica, donde poner a prueba conocimientos y destrezas y donde la inmediatez exige la acción. Experiencia que contribuirá a lograr una sólida formación sustentada tanto en lo cognitivo como en lo social. A diferencia de otras instancias de formación que ponen el énfasis en el aprendizaje académico o solo en el servicio social, al situar a nuestros alumnos en una metodología de aprendizaje-servicio, se pone el acento en los dos términos que constituyen esta expresión. Respecto de los alumnos agrotécnicos de las dos instituciones 


\section{consideramos impostergable sumar alumnos en trabajos de investigación- extensión de fuerte contenido e impacto social}

formoseñas con las que trabajamos, se aspira a que éstos sean multiplicadores de innovaciones al formarse y participar en el proyecto.

Para indagar acerca de la impronta que el trabajo supuso para los alumnos de la Facultad de Ciencias Veterinarias recurrimos a sus reflexiones, a las devoluciones escritas que ellos hicieron después de viajar a la localidad de El Colorado en los últimos seis años (2011-2016).

Al utilizar lo manifestado por los alumnos, lo por ellos vivido, la palabra escrita o enunciada son fuentes de datos cualitativos, y las expresiones vertidas tras los viajes son un material idóneo para hacernos de esa información.

Lo expresado se constituye así en "un reflejo de la subjetividad del emisor y revela su actitud, su sentir, su reflexión ante lo vivido", y se definen en esta función las relaciones internas y su autor. Las palabras de los actores fueron agrupadas alrededor de cinco ejes principales: aportes a la formación profesional, conocimiento de una realidad distinta, constitución de redes, posibilidad de hacer social y hacer del campo profesional.

\subsection{Aportes a la formación profesional}

"El hecho de haber viajado formando parte del proyecto me resultó una experiencia muy gratificante, en la cual sentí que la universidad devolvía, en parte, el aporte realizado por los ciudadanos para mantener viva nuestra institución.

Creo necesarios estos tipos de proyectos en los cuales los alumnos nos ponemos de cara a diferentes problemáticas del cotidiano ambiente rural, al poder ubicarnos en el mismo, ver, tocar, envolvernos en el día a día y oír, a través de las voces de sus actores (llámese propietario de un establecimiento, peón, contratista, estudiante de una escuela agrotécnica, etc.), su manera de realizar los trabajos que llevan a cabo e intercambiar conocimientos a través de la discusión y el trabajo conjunto."

"En cuanto a la transferencia de conocimientos, la verticalidad de nuestras exposiciones se "horizontaliza" al dar lugar a la discusión y participación activa de las personas a quienes van dirigidas las mismas; pudiendo el expositor, tanto en la parte práctica como en la teórica, incorporar conceptos aportados por quienes conocen la realidad local, al compartir sus experiencias, lo cual genera una bidireccionalidad en la transferencia del conocimiento."

"El viaje fue hermoso grandioso vine llena de muchas cosas, conocimientos e ideas, aprendí mucho de ellos y de ustedes me parece un gran trabajo e importante, estoy entusiasmada con muchísima ganas de seguir colaborando y participando. Esta sociedad nos necesita y hay mucho trabajo por hacer."

"Finalmente, quiero agradecer a todos los docentes y no docentes que hicieron posible la realización del viaje y espero que lo conserven con los años que vienen, ya que lo considero un complemento fundamental en la formación de un médico veterinario integral."

\subsection{Conocimiento de una realidad distinta}

"Escucharlo decir en cada frase (en referencia a lo manifestado por un productor) que lo pone muy contento el progreso de la cooperativa, el que más gente se sume, hace que uno se entusiasme y se convenza cada vez más que vamos por el camino correcto. $Y$ en resumidas cuentas es eso, ver y creer en que se puede y hacerlo realidad."

"Con respecto al viaje a Formosa, tengo que admitir que cada vez que voy, vuelvo con más ganas de viajar nuevamente, desde mi primer viaje siempre volví muy entusiasmada y con ganas de que más compañeros puedan viajar y conocer esa 'otra realidad', agradezco tener la oportunidad de conocer."

"Me gustó mucho ir y conocer la forma de vivir de las personas, los recursos que tienen y como los aprovechan."

"Pensé que me iba a encontrar con gente poco predispuesta al cambio y vi todo lo contrario, vi que tienen ganas de progresar, de ayudarse entre todos." 
"El hecho de vincularnos con otras regiones de nuestro país, nos lleva a conocerlo más y mejor. Más en el sentido de salir de nuestra realidad, que no es la realidad de todo nuestro gran país y mejor, porque podemos conocer el lugar, su ambiente, flora, fauna, personas y cultura; cuestiones todas que nos llevan a sentirnos parte, a acortar distancias, a conocer; conocer para querer, porque al final de cuentas no se puede querer aquello que no se conoce.

Realmente me siento muy agradecido por la oportunidad de haber formado parte de este proyecto. Espero podamos continuar con el mismo."

"Agradezco que me tomaran en cuenta para participar en este proyecto, que desde que llegué a Argentina ha sido una de las mejores experiencias, distinta y enriquecedora. El hecho de haber tenido la oportunidad de visitar y conocer personas de otra provincia rompe paradigmas y amplía el conocimiento cultural de lo que para mí era la República Argentina o simplemente un argentino." (Becario mexicano)

"En primer término, esta experiencia me enseñó la básica cuestión de que existe producción afuera de la llanura pampeana, diferente pero real. Las diversas materias cursadas a lo largo de la carrera no supieron darme un ejemplo tan sencillo del significado de la diversidad sociocultural y cómo esto, sumado al ambiente (clima + suelos), repercute sobre la manera de producir alimentos, como lo hizo este viaje al norte argentino. En segundo lugar, este viaje enseñó al grupo integrante en su totalidad la importancia que tiene la 'cultura del trabajo', una colección de tradiciones, costumbres y obligaciones que las provincias de la llanura pampeana perdieron hace rato, en las que fuimos criados, por lo tanto, muchos de nosotros quedamos mudos al observar tanta resistencia de esos pequeños productores a ser absorbidos por los grandes cinturones urbanos de nuestras provincias."

\subsection{Constitución de redes}

"Pienso que año tras año, éste siendo mi segundo año en el programa, va creciendo en forma asombrosa. Ya que no solo se cuenta con un grupo de docentes de excelente calidad humana y predisposición a transmitir su conocimiento sino que del otro lado nos encontramos con personas preocupadas y con ganas de tomar y dar también su conocimiento! Gratificante trabajo de generar un poco más de igualdad en este hermoso país, y a todos los involucrado como las escuelas, al INTA, productores y estudiantes que no solo nos permiten discutir las diferentes problemáticas, sino que nos hacen parte de su vida y costumbres."
"En cuanto a las actividades en general, creo que nos enriquecen tanto a ellos, como a nosotros, y también creo que queda mucho por trabajar, tanto hacia un lado como hacia el otro."

"En lo que a mí respecta me llena de emoción haber compartido ese tiempo con ustedes, creo que es necesario nutrirnos de información, estudiar y ser constantes con los que nos gusta y en ustedes pude notar eso. Se necesitan muchas ganas para asistir a clases en condiciones de lluvias, cuando los caminos están inundados y es difícil llegar. Pero para ustedes no es impedimento, y eso es de admirar."

\subsection{Posibilidad de hacer social}

"Chicos, quiero felicitarlos por el entusiasmo que ponen en cada actividad, no pierdan esas ganas de seguir avanzando a cada paso que dan, sigan preguntándose en cada actividad que podemos hacer para mejorar, apóyense en esos profesores de fierro que tienen la suerte de tener.

Crezcan y hagan crecer ese lugar hermoso donde viven, siempre con alegría y disfrutando cada momento.

Realmente admiro el respeto con el que hacen cada cosa, ese es un valor que da gusto ver.

Podría decirles muchas cosas más que no sería más que reiterar lo dicho antes.

Saben que cuentan con nuestro apoyo incondicional." (Dirigido a los alumnos de los dos colegios agrotécnicos)

"Sobre nuestra visita al tambero Jorge me pareció muy interesante e importante, me sirvió mucho para reflexionar la valoración, la humildad y la voluntad de conseguir alternativas nuevas para seguir adelante."

"En lo que respecta al viaje, fue una experiencia única. Fue muy refrescante conocer gente tan cálida y con tantos valores. Muchas veces nuestro ritmo no nos deja ver que si se puede parar y hacer las cosas con calma, como hacen los formoseños tan módicamente; también cabe destacar que su modelo productivo parece prometedor, aunque le queda un largo camino por recorrer, ya que es notoria la tendencia a la subsistencia que maquina dicho modelo."

"Me llego mucho la gente 'campesina' como la llaman allí, oír sus palabras como esa señora que decía que hacía 60 años vivía lo mismo y que cada vez estaban peor... creo que lo único que necesitan es un apoyo político y/o económico para crecer aún más y sería bueno que en un próximo viaje allá una respuesta desde ese lado." 
"Solía 'oír' en los medios de comunicaciones la existencia de realidades sociales diferentes, de pequeños productores, pero era solo eso, 'oírlo' y dejarlo pasar.

Vivimos en un mundo estandarizado, donde te imponen como vivir, que ver y creer. Pero cuando uno abre los ojos, ve y vive otra realidad, descubre que no todo lo que creemos importante lo es. Que se puede aprender a vivir y cosechar de la manera más simple, si uno pone verdaderamente su empeño y ganas de superarse.

Esto es lo que aprendí yo, desde mi reciente visión en este viaje a Formosa."

\subsection{Hacer del campo profesional}

"Después de este viaje me doy cuenta de que la producción a gran escala no es una ley que predomine en todo el país y que tanto la educación secundaria como la formación y actualización de pequeños productores es una tarea que los profesionistas como los médicos veterinarios, agrónomos, etc., tenemos la obligación de difundir y enseñar sobre todo entre nosotros mismos, lo digo porque como estudiante siento que nuestra formación está muy volcada del lado de la producción intensiva, los planes de estudio están repletos de una formación cada vez más cuadrada que nos da la idea de que las pequeñas producciones son ineficientes y poco competitivas en el mercado. Nos olvidamos que hay sectores de la sociedad que requieren los servicios de profesionistas que solucione los problemas y mejoren las condiciones de este tipo de producción." (Becario mexicano)

"Esta es la primera vez que participo de un proyecto de extensión y verdaderamente fue una experiencia positiva para mí. En principio me resulta importante rescatar la posibilidad de haber podido viajar para poder conocer el lugar de trabajo y que las personas de allí nos cuenten ellos mismos la situación en la que se encuentran y cuáles son sus problemáticas. Me resultó gratificante poder aportar algunos de los conocimientos que nos da la Facultad para de alguna manera colaborar con la sociedad. Quiero dar mi agradecimiento a ustedes los profesores, a mis compañeros y a los chicos y autoridades de las escuelas y productores de Formosa porque siento que todos pusieron lo mejor de cada uno y así pudimos compartir y aprender entre todos."

"Con respecto al viaje, sinceramente yo no sabía mucho de que se trataba, más que de ir a ayudar en diferentes actividades, estaba ansiosa, quise ir porque siempre tuve la inquietud de hacerlo y por diferentes motivos no lo hacía. Creo que este viaje para mí fue un antes y un después, ya sea por el hecho de ayudar, de aportar un granito de arena a esas personas, el hecho de verles las caras, que te escuchen, que se interesen, que quieran crecer, y ser nosotros parte de esa transformación, realmente me lleno el alma, me sentí muy bien.”

"Gracias por haber tenido la oportunidad de viajar y participar varias veces, me di cuenta en este viaje, primero, del avance del proyecto, de la importancia que tenemos como profesionales, y segundo del alcance que tiene nuestra profesión. Se puede, y como dije adelante de Jorge, me di cuenta de lo importante que es estar organizados y respetar al prójimo. Estar organizados y seguir, siempre tirando para adelante eso rescato, que podemos hacerlo. Vi el avance de Jorge después de casi dos años que no iba a su casa, las ganas con las que hace, dice y sueña todo. Gracias a vos por estimular este tipo de actividades, muy valorable para mí, me has abierto mis ojos, puedo ver más allá de lo que creía."

Damos cuenta de que, en función del criterio de saturación de datos o redundancia de los mismos, solamente hemos transcripto aquí una parte de las devoluciones elaboradas tras los viajes por los 60 estudiantes voluntarios del proyecto. $Y$ cabe mencionar que en los últimos seis años se realizaron 13 traslados con alumnos a El Colorado, lo que fue posible gracias a la obtención de subsidios.

\section{Evaluación de lo dicho}

Conocemos el valor de la palabra y su significado simbólico; también destacamos positivamente la acción de poder llevar a la escritura lo vivido, donde las letras no solo traducen lo hecho sino la implicancia que adquiere para el enunciante. Consecuentemente, consideramos que solo así se logran un sentido y una valía imposible de captar con otra estrategia metodológica.

Con referencia a la formación profesional, sabemos que el valor de esta experiencia respecto del diseño curricular quizás sea escaso. No así en cuanto a lo experimentado por los alumnos participantes, ya que ellos hablan de un antes y un después. Mencionan la importancia de situarse no solo como espectadores sino "el ser una parte activa de la problemática" hecho que les implica pensar, reflexionar y actuar, donde la búsqueda de soluciones los incumbe de una manera grupal.

Estos aprendizajes son holísticos ya que los introducen en situaciones reales donde deben poner en juego lo aprendido y cotejarlo con los saberes locales.

Cuando analizamos lo dicho en función de conocer una realidad distinta, los futuros veterinarios hablan de "esa otra realidad" no abordada en las aulas. "Salir de nuestra realidad", entre otras manifestaciones, nos indica no solamente de la fragmentación 
curricular sino que la universidad muchas veces mira hacia dentro de sí misma e ignora la sociedad y sus reclamos. Los voluntarios señalan la importancia de este tipo de proyectos, agradecen el haber podido participar y el deseo de que puedan hacerlo otros compañeros. Creemos que ello podría traducirse así:

"Las diversas materias cursadas a lo largo de la carrera no supieron darme un ejemplo tan sencillo del significado de la diversidad sociocultural y cómo esto, sumado al ambiente (clima + suelos), repercute sobre la manera de producir alimentos, cómo lo hizo este viaje". (Alumno)

También podemos mencionar que la conformación de redes es otro de los logros, hecho que se manifiesta cuando nuestros alumnos universitarios analizan los lazos establecidos con los alumnos de los dos establecimientos secundarios con los que trabajamos, cuando evalúan lo logrado por la Cooperativa o con técnicos y productores de la región, ya que el equipo docente es solo una de las partes del proyecto, puesto que trabajamos con una cooperativa, un instituto terciario, dos colegios agrotécnicos, personal del INTA y productores tanto de Cambio Rural como independientes.

Si analizamos lo manifestado respecto de la posibilidad de un hacer social, encontramos dichos que lo ejemplifican, como el de que antes era solo "oírlo y dejarlo pasar", ya que da cuenta de asumir una realidad y comprometerse.

Con relación al hacer del campo profesional, bastan las palabras de un alumno becario mexicano, quien nos acompañó en un viaje durante 2015:

"Que tanto la educación secundaria como la formación y actualización de pequeños productores es una tarea que los profesionistas como los médicos veterinarios, agrónomos, etc. tenemos la obligación de difundir y enseñar sobre todo entre nosotros mismos, lo digo porque como estudiante siento que nuestra formación está muy volcada del lado de la producción intensiva, los planes de estudio están repletos de una formación cada vez más cuadrada que nos da la idea de que las pequeñas producciones son ineficientes y poco competitivas en el mercado".

\section{A modo de cierre}

Concluimos que este tipo de actividades son intentos aislados dentro del curriculum de la Facultad y que, si bien este proyecto ha movilizado solo una parte de los alumnos de la carrera de
Ciencias Veterinarias, el hecho de que ellos participen de manera activa tanto en la planificación como en la concreción de las actividades y de que se comprometan las convierte en acciones cualitativas en su formación. Además, estos proyectos se constituyen en espacios que permiten el encuentro y el diálogo entre distintos actores.

Al decir de Milagros Rafaghelli (2013:22): "así comprendida, la extensión no es descripción sino acción intersubjetiva”, pero debemos establecer que para que este manifiesto se haga realidad es necesario posicionarnos como docentes en un paradigma distinto del de simple transmisor y utilizar metodologías de enseñanza y de aprendizaje que permitan conocer, reflexionar y hacer a nuestros alumnos universitarios.

No tenemos dudas de que las acciones de extensión encuadradas como Práctica Social Comunitaria, que promuevan aprendizajes significativos, se constituyen, de acuerdo con Socolovsky, en instrumentos que permiten "revisar contenidos, objetivos y modalidades de la enseñanza" (2015:12) y seguramente de esta manera se estará instando a la curricularización de la extensión en la universidad. 


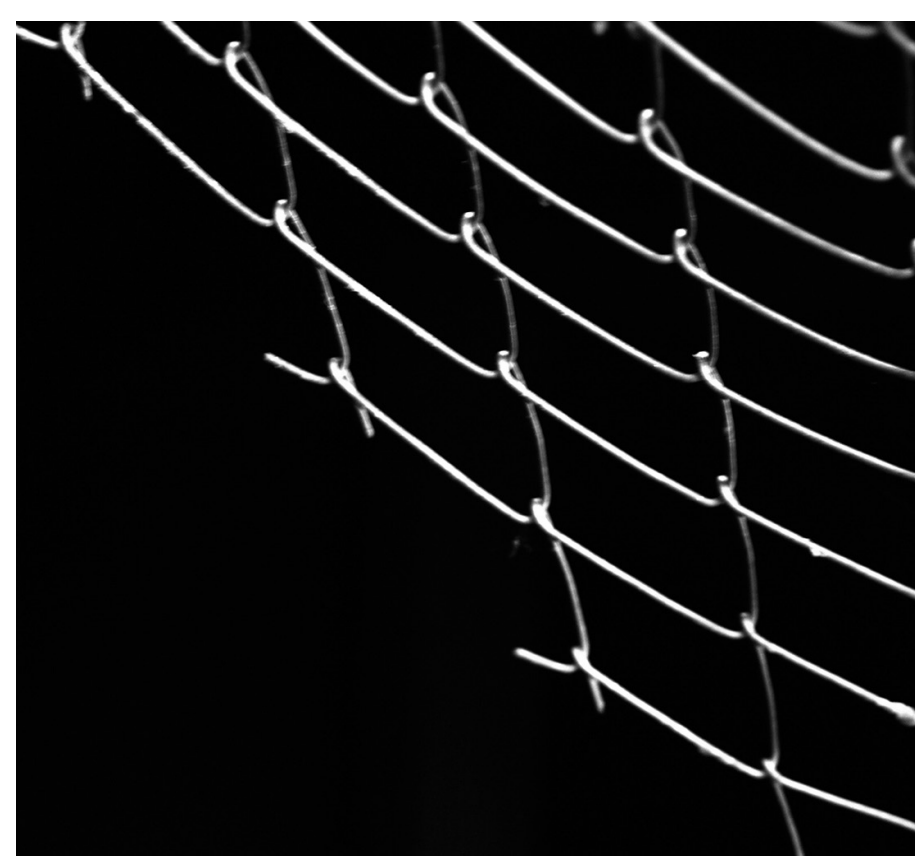

(C) Laura Hormaeche

\section{6}

los alumnos participantes mencionan

la importancia de situarse no solo como espectadores sino "el ser una parte activa de la problemática"

\section{Referencias bibliográficas}

Biolatto, R. (2005). El diseño curricular como posibilitador de la práctica profesional. El plan de estudios 2003, Facultad de Ciencias Veterinarias UNR. Tesis de Maestría inédita. UTN Regional Rosario.

Biolatto, R. (2011). Lo hecho en Santa Fe con pequeños tamberos. En Biolatto, R. (Coord.), Entendiendo a los pequeños productores lecheros. Un acercamiento a su problemática. Rosario: UNR Editora.

Camilloni, A. (2013). La inclusión de la educación experiencial en el currículo universitario. En Menéndez, G. y otros, Integración, Docencia y Extensión. Otra forma de aprender. Santa Fe: Ediciones UNL.

Chiroleu, A.; lazzeta, O.; Vora, C.; Díaz, C. (2001). La política universitaria argentina de los 90: Los alcances del concepto de autonomía. Education Policy Análisis Arribes, 9 (22)

Contreras, J. (1997). La autonomía del profesorado. Madrid: Morata.

Dasso, L. (2011). Universidad y Extensión. Hacia un cambio de paradigma. En Biolatto, R. (Coord.), Entendiendo a los pequeños productores lecheros. Un acercamiento a su problemática. Rosario: UNR Editora.

De Alba, A. (2004). "Crisis estructural generalizada: sus rasgos y sus contornos sociales". En Libro de Ponencias // Congreso Internacional de Educación. Santa Fe: Ediciones UNL.

Erreguerena, F.; Nieto, G.; Touza, R.; Hernando, G. (2015). La Extensión Universitaria en la Universidad Nacional de Cuyo (2008-2014). Programa de Proyectos "Profesor Maurio A. López": Una práctica educativa desde el compromiso social de la Universidad. En Castro, J.; Oyarbide, F. (Comps.), Los caminos de la extensión en la Universidad Argentina. La Pampa: UNLPam.

Pérez Zaballa, L. (2007). Pertinencia y Extensión Universitaria en el contexto de la nueva universidad cubana. Pedagogía Universitaria, XII(I). Disponible en: http://cvi. mes.edu.cu/peduniv/index.php/peduniv/article/view/395/386

Rafaghelli, M. (2013). La dimensión pedagógica de la extensión. En Menéndez, G. y otros, Integración, Docencia y Extensión. Otra forma de aprender. Santa Fe: Ediciones UNL.

Rosemberg, F. y Casas Olascoaga, R. (1991). Ciencias Veterinarias y sociedad: Reflexiones sobre el paradigma profesional. Educación Médica y Salud, 25(3), 333-345.

Tapia, M.N. (2010). Aprendizaje y servicio solidario. En el sistema educativo y las organizaciones juveniles. Buenos Aires: Ciudad nueva.

Reyes Sánchez, O.; Plascencia Jorquera, A. (1993). Propuesta metodológica de innovación para obtener un perfil profesional del médico veterinario zootecnista y estrategias de enseñanza-aprendizaje eficientes y económicas. Encuentro iberoamericano de expertos en educación de la Medicina Veterinaria y Zootécnica. La Habana.

Socolovsky, Y. (2015). Prólogo. En Antequera, M.; Chevasco, M.; Craparo, R.; Serra, M., Articulación Social y Prácticas Sociocomunitarias. Un desafío para la Universidad Pública. Rosario: UNR Editora. 